\title{
COMPETÊNCIAS INFORMACIONAIS PARA UTILIZAÇÃO DA ANÁLISE BIBLIOMÉTRICA EM EDUCAÇÃO E EDUCAÇÃO ESPECIAL
}

\author{
Maria Cristina Piumbato Innocentini Hayashi \\ Márcia Regina da Silva \\ Carlos Roberto Massao Hayashi \\ Amarílio Ferreira Júnior \\ Leandro Innocentini Lopes de Faria
}

\section{RESUMO}

No atual contexto da sociedade do conhecimento, os saberes produzidos pelas transformações tecnológicas tornam-se mais acessíveis através do uso das tecnologias da informação e comunicação. Fundamentadas nos princípios da interdisciplinaridade e na superação da visão fragmentada do conhecimento e com base em pesquisas concluídas nas áreas de Educação e Educação Especial foram identificadas as competências informacionais necessárias para a gestão de informações científicas e tecnológicas. As pesquisas utilizaram metodologicamente a análise bibliométrica para avaliar: a produção científica brasileira na base de dados Francis do INIST/CNRS, França; e a produção científica dos docentes do Programa de Pós-Graduação em Educação Especial/UFSCar. Utilizou-se o quadro teórico composto dos históricos da Educação e Educação Especial no Brasil; da abordagem sociológica da comunidade científica; dos aspectos conceituais sobre banco de dados e estudos bibliométricos; da revisão de literatura sobre competências informacionais. A análise dos dados obtidos nas pesquisas e a reflexão a partir do quadro teórico utilizado permitiram identificar as competências e habilidades na realização dessas pesquisas: contexto de produção da informação; realizar operações de acesso, busca, avaliação, seleção e recuperação das informações; domínio de ferramentas automatizadas para reformatação e importação de dados, análise e síntese das informações; habilidades para validação, comunicação e disseminação dos resultados.

\section{PALAVRAS-CHAVE}

Competência informacional; Análise bibliométrica; Informação para educação; Educação especial 


\title{
INFORMATION COMPETENCIES FOR THE BIBLIOMÉTRIC ANALYSIS IN EDUCATION AND SPECIAL EDUCATION
}

\begin{abstract}
In the current context of the knowledge society, the technological transformations produce knowledge that characterize the contemporary world through the use of the information and communication technology, based on the principles of the interdisciplinar and the overcoming of the fragmented vision of the knowledge. Based in research concluded in the areas of Education and Special Education the necessary informational competency for the management of the scientific and technological information has been identified. In this research, the automatized bibliometric analysis was used as methodological resource to analyze: the Brazilian scientific production in database Francis of the INIST/CNRS, France; and the scientific production of the professors of the Graduates Program of Special Education/UFSCar. The starting point was the examination of the used theoretical constructs: historical of the Education and the Special Education in Brazil; sociological approach of the scientific community; conceptual aspects on database and bibliometrics studies; revision of literature on informational competency. The analysis of the data of the research and the reflection on the theoretical constructs had allowed to identify the competencies and abilities necessary in this research: context of production of the information; operations of access, search, evaluation, election and retrieval of the relevant information in texts or databases; domain of tools automatized for reformatation and importation of data, analysis and synthesis of the information; abilities for validation, communication and dissemination of the results.
\end{abstract}

\section{KEYWORDS}

Information literacy; Bibliometric analysis; Information for education; Special education 


\section{INTRODUÇÃO}

O século XXI é chamado de "sociedade do conhecimento" devido aos inúmeros meios de difusão das informações e da valorização despendida àqueles que têm acesso a essas informações e sabem utilizá-las em seu cotidiano. Nunca tivemos tantos meios de difusão de informações: impressos (jornais, revistas, livros, etc.), televisivo, radiofônico e digital. Nunca se produziu tanta informação. Podemos afirmar, com isso, que é praticamente impossível dizer que existe um assunto que não tenha sido tema de um trabalho, científico ou não.

O problema não é mais a falta de informação, mas sim, a forma de recuperação dessa informação. Por um lado nós temos a informação desejada, por outro lado não temos como filtrar todo esse volume de informação de forma pragmática e eficiente. A questão que se coloca é: Quais as competências necessárias para a gestão da informação na sociedade atual?

De forma oposta a essa tendência, o mundo moderno desemboca em uma forma de segregação e desigualdade configurada por aqueles que têm acesso à informação e aqueles que estão distantes dela. Está surgindo um novo tipo de analfabeto: o analfabeto digital, que, a despeito de toda tecnologia disponível, não está tendo acesso às informações importantes para sua vida. Então, outro problema social se coloca: Como incluir tais pessoas?

É nesse sentido que a Ciência da Informação pode ter importante papel na evolução da sociedade da informação. De nada adianta possuirmos inúmeros meios de difusão da informação se as poucas pessoas que tem acesso não estão conseguindo utilizálas de forma correta. Essa área tem por objetivo racionalizar o uso da informação e tornar esse uso acessível ao maior número de pessoas de forma eficiente. Com base nesse enfoque o artigo tem como objetivo identificar as competências informacionais necessárias para a utilização da análise bibliométrica enfocando dois estudos que recorreram a essa ferramenta para produzir indicadores na área de Educação (HAYASHI, 2004) e Educação Especial (SILVA, 2004). 
Hayashi (2004) objetivou realizar um levantamento e análise bibliométrica da produção científica brasileira sobre a temática Educação, presente em uma base de dados internacional, a Base de Dados Francis ${ }^{\circledR}$, do Institut de IInformation Scientifique et Technologique - INIST do Centre National de la Recherche Scientifique - CNRS, na França, como forma de contribuir para a compreensão da produção científica em Educação no Brasil.

Silva (2004) também utilizou a análise bibliométrica para avaliação da produção científica dos docentes do Programa de Pós-Graduação em Educação Especial da Universidade Federal de São Carlos.

Estes dois trabalhos, cada qual a sua maneira, contribuem para a discussão sobre a produção científica brasileira, pois utilizam mecanismos de avaliação da quantidade e qualidade desta produção. A produção científica brasileira é relativamente baixa em relação à produção científica mundial, mas nos últimos anos foi possível observar um avanço da produção científica, concentrada nos cursos de pós-graduação do País.

A avaliação da produção científica é de fundamental importância para medirmos a qualidade das pesquisas científicas nos programas de pós-graduação. Para Oliveira (1992, p.239), "a avaliação deve ser um dos elementos principais para o estabelecimento e acompanhamento de uma política nacional de ensino e pesquisa, uma vez que permite um diagnóstico das potencialidades das instituições acadêmicas". A produção científica faz parte de um ciclo que percorre a geração de idéias, o desenvolvimento da pesquisa e a comunicação. Essa comunicação é que impulsiona os progressos científicos, tecnológicos e culturais do País. Moura (2002, p.34) define produção científica como um:

vetor importante para a consolidação do conhecimento nas áreas do saber. A universidade é, portanto, o locus por excelência onde essa produção é gerada, advinda das pesquisas e estudos desenvolvidos no meio acadêmico, nos vários campos do conhecimento.

A avaliação da produtividade científica é um elemento importante para o estabelecimento de políticas nacionais de ensino e pesquisa, pois permite diagnosticar as 
potencialidades de determinados grupos e/ou instituições. Para que seja realizado o diagnóstico, é necessário utilizar técnicas específicas de avaliação que podem ser tanto qualitativas como quantitativas.

A expansão da ciência e da tecnologia trouxe a necessidade de avaliação e acompanhamento do desenvolvimento e dos avanços alcançados pelas diversas áreas do conhecimento. Além da bibliometria, existem diversas formas de medição voltadas para avaliar a ciência e os fluxos de informação, dentre as quais podemos citar: a cientometria, a informetria e a webmetria. Cada uma dessas técnicas quantitativas busca enfoques diferentes para medir a difusão do conhecimento científico.

O princípio da bibliometria é analisar a atividade científica ou técnica pelo estudo quantitativo das publicações. Esta concepção da bibliometria levou Rostaing (1997) a adotar a abordagem de que a bibliometria é a aplicação dos métodos estatísticos ou matemáticos sobre o conjunto de referências bibliográficas.

Para Macias-Chapula (1998, p.134), a bibliometria pode ser definida como:

o estudo dos aspectos quantitativos da produção, disseminação e uso da informação registrada. A bibliometria desenvolve padrões e modelos matemáticos para medir esses processos, usando seus resultados para elaborar previsões e apoiar tomada de decisões.

O uso da bibliometria pode ser vantajoso nos seguintes aspectos: na contribuição às avaliações de pesquisa na universidade; na avaliação de grupos de pesquisa da mesma área; na avaliação da contribuição de pesquisadores para determinada área, e na classificação entre instituições, etc.

\section{TRANSFORMAÇÕES TECNOLÓGICAS E CONVERGÊNCIAS NA INDÚSTRIA DO CONHECIMENTO}

Observamos que nos últimos anos tem havido um aumento da discussão sobre as mudanças de paradigma na área científica, tecnológica, econômica, política e social, devido às transformações tecnológicas que modificaram totalmente o processo de 
comunicação e informação. Verifica-se a existência de um paradigma emergente de produção de bens e serviços e de organização de atividades em geral baseado na utilização intensiva das Tecnologias da Informação e da Comunicação.

O impacto da utilização das novas tecnologias contribuiu para que as organizações se direcionassem para o conhecimento. Dessa forma, a sociedade impõe o contínuo desenvolvimento de novos saberes e competências, tendo como principal meta deter o conhecimento avançado sobre as tecnologias de informação e comunicação que ocupam o centro da dinâmica de inovações.

Não é possível imaginar mais a vida sem todos os aparatos tecnológicos que nos cercam. Dominar essa tecnologia tornou-se requisito básico para o aprendizado nesta sociedade do conhecimento.

Hoje, a informática representada pelos computadores, softwares e interfaces, as telecomunicações, representada pelas diversas redes (TV a cabo, telefonia e satélites) e o conteúdo, representado pelos bancos de dados, filmes, músicas, imagens e propaganda, formam a multimídia interativa que, por sua vez, representa a indústria do conhecimento. Ou seja, a informática, as telecomunicações e o conteúdo, são elementos que, combinados, garantem a existência e evolução da sociedade atual, que se caracteriza pela "aprendizagem ao longo da vida” e se preocupa cada vez mais com a importância de se converter informação em conhecimento. Além do acesso, é importante a assimilação crítica da informação. É preciso dar sentido a este “mar” de informações que está em todo lugar.

Atualmente vivemos em uma sociedade em rede graças às tecnologias de informação e comunicação disponíveis. Nesta sociedade em rede, podemos identificar as seguintes características: a interatividade, caracterizada como uma novidade única; a interdisciplinaridade é a marca distintiva da Internet, ou pode-se mesmo dizer, que significa uma nova forma de construção do conhecimento; o hipertexto - um dos paradigmas básicos da teia mundial; multimídia - tendência de utilização de múltiplas mídias - a informação pode ser comunicada por múltiplos meios: textos, imagens, sons, filmes, animações, etc.; digitalização das imagens e textos em um ambiente de hipertexto; o compartilhamento das informações. 


\section{ESTUDOS BIBLIOMÉTRICOS NO CONTEXTO DA EDUCAÇÃO E EDUCAÇÃO ESPECIAL: DUAS EXPERIÊNCIAS}

As transformações que ocorrem atualmente em todas as áreas do saber são influenciadas pela presença forte das novas tecnologias de informação e tecnologia. Nas áreas de Educação Especial e Educação o quadro atual impõe:

- novas concepções de práticas educativas e informacionais - os paradigmas epistemológicos clássicos, incapazes de compreender e enfrentar desafios da contemporaneidade foram postos em discussão no atual quadro histórico-social;

- abordagens teórico-metodológicas inovadoras - para a compreensão e o enfrentamento das complexas inter-relações entre Informação e Educação nos tempos presentes;

- superar a atual dicotomia entre área de conhecimento e campo de atuação, promovendo práticas interdisciplinares cujos resultados contribuam para a modificação do cenário atual da pesquisa em Educação Especial no país.

A partir desse novo contexto é que as duas experiências realizadas nas áreas de Educação e Educação Especial ganham novos contornos com a utilização de metodologias que utilizam ferramentas tecnológicas: os estudos bibliométricos.

Para além das discussões e polêmicas que costumam cercar os métodos quantitativos, tendo em vista o papel essencial das ferramentas matemáticas e as estatísticas neste contexto, esclarece-se que a análise bibliométrica, embora esteja associada à ciência da ciência e a seu positivismo, se funda em análises qualitativas como as que foram desenvolvidas pelas correntes mais recentes da antropologia ou da história social das ciências. As estatísticas não constituem um fim em si, mas são mobilizadas para analisar a dimensão coletiva da atividade de pesquisa e o processo dinâmico da construção de conhecimentos (SILVA, 2004). 
A análise bibliométrica é utilizada em pesquisas nas diversas áreas do conhecimento para a obtenção de indicadores de ciência e envolve a aplicação de conhecimentos que ultrapassam as habilidades em somente aplicar o método. Nesse sentido, apresentamos na Figura 1 um esquema da organização dos estudos de Hayashi (2004) e Silva (2004).

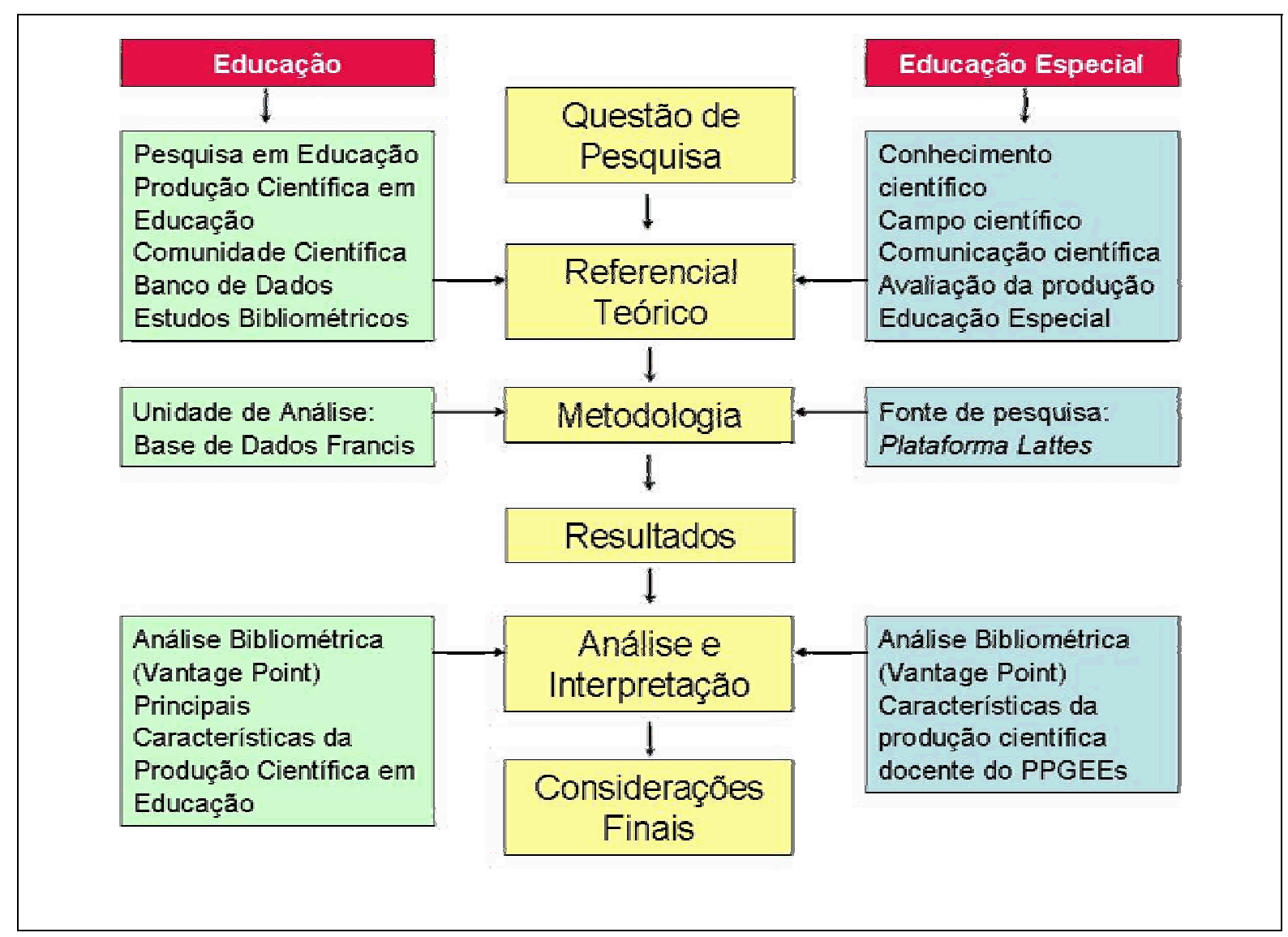

FIGURA 1 - Esquema da organização dos estudos de Hayashi (2004) e Silva (2004).

É possível verificar que os dois pesquisadores percorreram caminhos parecidos para a obtenção dos resultados. Partiram de uma problemática, momento crucial para direcionar a pesquisa; e passaram para um segundo momento, no qual é feito todo o embasamento teórico da pesquisa. Na metodologia foram descritos o ambiente da pesquisa, os recursos utilizados para coleta de dados e a operacionalização desses recursos. Os resultados foram obtidos mediante a aplicação da análise bibliométrica automatizada, com a 
utilização em ambos os trabalhos, do software Vantage Point. Este software, através de recursos de relacionamento de dados, permite a obtenção de indicadores de acordo com os objetivos do pesquisador. Já a análise e interpretação dependem do referencial teórico e dos resultados para ser realizada.

As duas pesquisas seguiram os padrões recomendados para a elaboração de trabalhos científicos, porém, o que nos interessa, é deter-nos na análise bibliométrica para identificarmos as competências informacionais necessárias para a aplicação deste método.

Dessa forma, no contexto desse artigo, conceituaremos os termos "competência" e "competência informacional".

\section{COMPETÊNCIA: ABORDAGENS E FORMAS DE CLASSIFICAÇÃO}

Seguindo a lógica capitalista de produção e as transformações ocorridas na sociedade da informação, o mercado passa a não mais falar em qualificação profissional para exercer uma função e, sim em competência. Competência é vista como as qualidades mais completas que um indivíduo possui para desenvolver uma atividade.

Miranda (2004, p.114) identificou quatro períodos da evolução do termo competência:

- Anos 70: neste período o termo propriamente dito não era usado, mas foi marcado pelo surgimento das primeiras noções fundamentais, alterando os valores do modelo de trabalho vigente: autonomia, expressão individual e responsabilidades;

- Primeira parte dos anos 80: a prática ainda se associava ao posto de trabalho e qualificação profissional, mas a temática competência já se destacava, evidenciando mais uma noção fundamental: a delegação de responsabilidade;

- Primeira parte dos anos 90: O reconhecimento das competências iniciou-se a procura de métodos e foram realizadas as primeiras pesquisas; 


\section{Área temática: Biblioteconomia \& Ciência da Informação}

- Final dos anos 90: desconexão entre qualificação (para o emprego) e competência. A competência toma lugar no contexto da gestão de recursos humanos e se informatiza, tomando também um lugar crescente dentro do debate social.

As competências, diferentemente da qualificação, começava a ser entendida como competências individuais independente da função exercida. A noção de competência ultrapassa o posto de trabalho e se foca nas competências gerais do indivíduo.

Hoje, a literatura define competência com ênfase diferente, de acordo com a área em questão. O termo competência pode se referir à capacidade para tomar decisões adequadas em âmbito definido, ou seja, aplicar seus conhecimentos para resolução de problemas (HARLEN, 1989; MAMEDE; PENAFORTE, 2001). Ainda neste contexto, a competência é definida como a colocação de recursos em uma situação prática. É a faculdade de mobilizar redes de atores em volta das mesmas situações, de compartilhar desafios, de assumir áreas de responsabilidade (ZARIFIAN, 2001).

As competências podem ser abordadas em diferentes níveis, tais como:

- Competências organizacionais - como o conjunto de habilidades e competências individuais nos processo decisórios da organização, constituindo uma fonte de vantagem competitiva (UBEDA, 2003, p.34)

- Competências individuais - envolvem o estoque de conhecimentos, habilidades e atitudes de um indivíduo, além dos resultados, produção e entrega decorrentes de sua mobilização, incorporando-os ao negócio da organização (UBEDA, 2003, p.37).

- Competências-chave - representam a aprendizagem coletiva da organização, especialmente sobre como coordenar diversas habilidades produtivas e integrar múltiplas correntes de tecnologia (PRAHALAD; HAMEL, 1990, p.82).

Zarifian (2001, p.115) considera que são os diferentes conteúdos concretos das competências que as diferenciam. É possível caracterizar competência como: 
- Conceito multidimensional - principais atributos são: iniciativa, responsabilidade, inteligência prática; conhecimentos adquiridos, transformações, diversidade; mobilização dos atores, compartilhamento.

- Centra atenção sobre o indivíduo e suas qualidades.

Podemos observar na figura 2 as três dimensões da competência definidas por Durand (2000).

\section{Conhecimentos}

\section{Informação \\ Saber o quê e por quê fazer

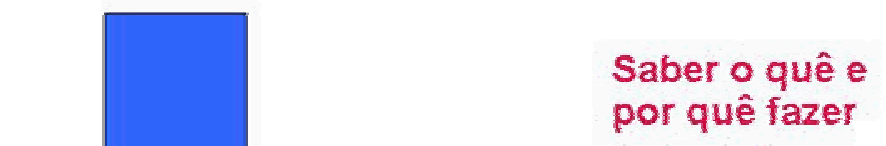

Atitudes

- Técnica

- Capacidade

Saber como fazer
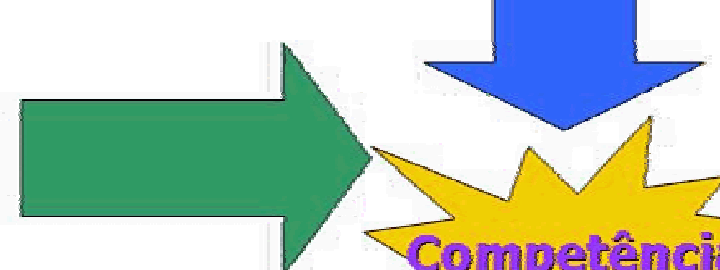

Competênciá Z.
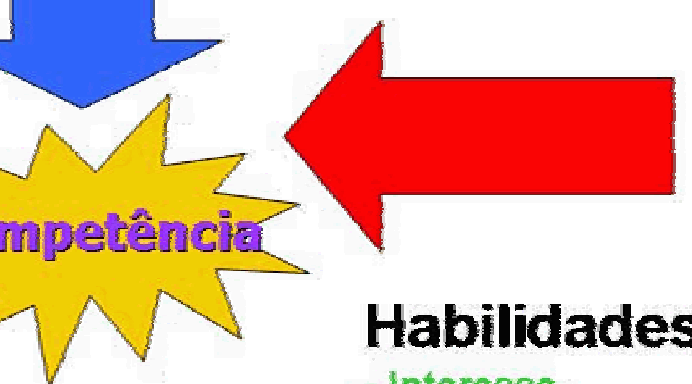

Habilidades

- Interesse

- Determinação Querer fazer

FIGURA 2 - As três dimensões da competência definidas por Durand (2000)

\section{COMPETÊNCIA INFORMACIONAL}

A competência informacional é um tipo de competência específica: a competência para se lidar com a informação. Segundo Miranda (2004, p.118), competência informacional é: 
o conjunto das competências profissionais, organizacionais e competênciaschave que possam estar ligadas ao perfil de um profissional da informação ou de uma atividade baseada intensivamente em informação. Pode ser expressa pela expertise em lidar com o ciclo informacional, com as tecnologias da informação e com os contextos informacionais.

O termo competência informacional foi utilizado como Information literacy, inicialmente nos EUA para designar habilidades ligadas ao uso da informação eletrônica (CAMPELLO, 2003). Dudziak (2003) discutiu o termo Information literacy abordando sua função educacional. Propôs ainda, diversas possibilidades de tradução do termo: alfabetização informacional, letramento, literacia, fluência informacional, competência em informação.

As competências informacionais estão ligadas ao processo informacional, processo este estudado pela Ciência da Informação. Essas competências estão ligadas à tecnologia da informação, ao ciclo e análise da informação.

\section{COMPETÊNCIAS PARA REALIZAR ANÁLISES BIBLIOMÉTRICAS}

Ao analisar os trabalhos de Hayashi (2004) e Silva (2004), identificamos as seguintes competências para a realização de análises bibliométricas:

a) conhecimento do contexto de produção da informação

É preciso antes de qualquer coisa, definir o campo do conhecimento. Hayashi (2004) definiu neste momento a área de Educação, e Silva (2004) a área de Educação Especial. Ambos realizaram o "estado da arte" das áreas pesquisadas, com base nos conhecimentos obtidos e estabeleceram os descritores que melhor caracterizam as áreas.

b) capacidade para realizar operações de acesso, busca, avaliação, seleção e recuperação das informações relevantes em textos ou bases de dados

Campello (2003, p.32) fala em três competências para a perspectiva da competência informacional: competência para lidar com a informação; informação para a aprendizagem independente e; informação para a responsabilidade social. Neste momento 
observa-se que é preciso o conhecimento de algumas técnicas da Ciência da Informação para o sucesso da análise bibliométrica. As habilidades desejáveis são: domínio das diferentes ferramentas para pesquisa na Internet (sites, ferramentas de busca e bases de dados); manuseio da "linguagem documentária" para estabelecer estratégias de busca de informações; refinar os dados obtidos para avaliação das informações; estabelecer critérios para selecionar e recuperar as informações relevantes em relação aos objetivos da pesquisa.

No trabalho de Hayashi (2004) e Silva (2004) foi preciso o conhecimento das bases utilizadas, Francis e Plataforma Lattes, respectivamente, para definir as estratégias de recuperação dos dados. Também foi necessário conhecer as terminologias da área de Educação e Educação Especial para a definição das palavras-chave que seriam utilizadas para a recuperação dos dados. O objetivo dos trabalhos deve estar em foco a todo instante, de modo a estabelecer critérios para selecionar as informações relevantes em conformidade com o objetivo proposto.

c) domínio de ferramentas automatizadas para reformatação e importação de dados, análise e síntese das informações obtidas

Como já foi dito anteriormente, ambos os trabalhos utilizaram a análise bibliométrica automatizada. Nesse sentido, verificou-se que antes de optar por este caminho foi importante conhecer as ferramentas a serem utilizadas para o desenvolvimento da pesquisa. No trabalho de Hayashi (2004), foi utilizado o software Infotrans para a importação dos dados da Base de Dados Francis para o software Vantage Point. O Infotrans é um software para conversão de arquivos procedentes de quaisquer bases de dados ou de tratamento de texto para um novo formato a definir. Ao proceder a reformatação dos dados, o Infotrans prepara-os para a análise automática, ou seja, tem como finalidade eliminar todos os campos que não serão analisados, assim como retirar sinais e termos desnecessários, limpando a base de trabalho.

Ao optar para a utilização do Infotrans, Hayashi (2004) preocupou-se com a padronização dos dados e com a melhor qualidade na utilização do Vantage Point. Já Silva (2004), optou por padronizar os dados utilizando ferramentas simples do Word. Neste caso, a quantidade de dados analisados permitiu esta escolha. 
Na seqüência, com os dados já no formato bibliométrico desejado, os autores utilizaram o software Vantage Point para o tratamento bibliométrico. Os dados obtidos foram transportados para o Excel para elaboração de tabelas e gráficos. A análise bibliométrica permite realizar relacionamentos entre os diversos campos selecionados da base de dados, bem como traçar a matriz de relacionamentos entre autores, áreas de conhecimento e outros.

Em toda etapa da análise bibliométrica eletrônica observou-se a importância de conhecer e dominar as ferramentas de suporte. As habilidades desejáveis e necessárias são: conhecimento dos principais softwares para reformatação e importação de dados (Infotrans e Word); utilização de ferramentas de análise bibliométrica automatizada (Vantage Point); elaboração de tabelas e gráficos para síntese e apresentação das informações (Excel).

d) habilidades para validação, comunicação e disseminação dos resultados obtidos.

Após obter os resultados, em ambos os trabalhos foi preciso recorrer ao referencial teórico para a elaboração de categorias de análise. É importante destacar, que o pesquisador precisar conhecer a área pesquisada, e se apropriar do conhecimento adquirido no referencial teórico para saber interpretar os resultados obtidos. Somente assim, será possível estabelecer relacionamento entre os dados e construir os indicadores.

Com base nesses estudos podemos ainda destacar como competência, a habilidade em elaborar trabalhos científicos, tendo como objetivo a comunicação científica. Uma pesquisa só alcançará reconhecimento se for divulgada. A publicação dos resultados é o momento de submeter a pesquisa à crítica externa e contribuir com o desenvolvimento da ciência.

Resumindo, para a realização dos trabalhos são necessárias habilidades e competências que podem ser traduzidas nas seguintes etapas: recorrer ao referencial teórico para elaborar categorias de análise; estabelecer relacionamentos entre os dados obtidos; construir indicadores dos resultados obtidos; elaborar trabalhos científicos (artigos, livros, comunicações etc.) para divulgação e disseminação dos resultados; submeter os resultados à crítica externa. 


\section{CONSIDERAÇÕES FINAIS}

O desenvolvimento de competências informacionais para a utilização da análise bibliométrica é necessário para qualquer pesquisador, independente da área a qual se aplicará este método de avaliação científica. De maneira geral, pode-se dizer que as competências identificadas nesse artigo estão presentes em outras atividades ligadas à gestão da informação e do conhecimento.

\section{REFERÊNCIAS}

CAMPELLO, B. O movimento da competência informacional: uma perspectiva para o letramento informacional. Ciência da Informação, Brasília, v.33, n.3, p.28-37, set./dez. 2003.

DUDZIAK, E. A. Information literacy: princípios, filosofia e prática. Ciência da Informação, Brasília, v.32, n.1, p.23-35, 2003.

DURAND, J. S. L'alchimie de la compétence. Revue Française de Gestion, n.127, p.84102, jan./fév. 2000.

HARLEN, W. Enseñanza y aprendizaje de las Ciencias. Madrid: Morata, 1989.

HAYASHI, C. R. M. Presença temática da educação na base de dados Francis ${ }^{\circledR:}$ uma abordagem bibliométrica. 175f. 2004. Dissertação (Mestrado em Educação) - Universidade Federal de São Carlos.

MACIAS-CHAPULA, C. A. O papel da informetria e da cienciometria e sua perspectiva nacional e internacional. Ciência da Informação, Brasília, v.27, n.2, p.134-140, maio/ago. 1998.

MAMEDE, S.; PENAFORTE, J. Aprendizagem baseada em problemas. São Paulo: Hucitec, 2001.

MIRANDA, S. V. Identificando competências informacionais. Ciência da Informação, Brasília, v.33, n.2, p.112-122, maio/ago. 2004.

MOURA, A. M. S. de; MATTOS, C. V. de; SILVA, D. C. da. Acesso e recuperação da produção científica pela biblioteca universitária: os Anais de Eventos. In: SEMINÁRIO NACIONAL DE BIBLIOTECAS UNIVERSITÁRIAS, 12., Rio de Janeiro, 2002. Anais... Rio de Janeiro: UFF, 2002. 
OLIVEIRA, A. C.; DÓREA, J. G.; BOMENE, S. M. A. Bibliometria na avaliação da produção científica da área de nutrição registrada no Cibran: período de 1984-1989. Ciência da Informação, Brasília, v. 21, n.3, p.239-242, set /dez. 1992.

PRAHALAD, J. K.; HAMEL, G. The core competence of the corporation. Harvard Business Review, p. 79-91, May/Jun., 1990.

ROSTAING, H. La bibliométrie et ses techniques. Toulouse: Sciences de la Société; Marseille: Centre de Recherche Rétrospective de Marseille, 1997.

SILVA, M. R. da. Análise bibliométrica da produção científica docente do Programa de Pós-Graduação em Educação Especial da UFSCar: 1998-2003. 168f. 2004. Dissertação (Mestrado em Educação Especial) - Universidade Federal de São Carlos.

UBEDA, C. L. A gestão de competências em uma empresa de pesquisa e desenvolvimento. 117f. 2003. Dissertação (Mestrado em Engenharia de Produção) Escola de Engenharia de São Carlos, Universidade de São Paulo, São Carlos.

ZARIFIAN, P. Objetivo competência: por uma nova lógica. São Paulo: Atlas, 2001. 
MARIA CRISTINA PIUMBATO INNOCENTINI HAYASHI

Professora adjunto do Departamento de Ciência da Informação I

Programa de Pós-Graduação em Educação Especial da

Universidade Federal de São Carlos

E-mail: dmch@power.ufscar.br

MÁRCIA REGINA DA SILVA

Bibliotecária chefe do Centro Universitário Barão de Mauá

E-mail: marciars@baraodemaua.br

CARLOS ROBERTO MASSAO HAYASHI

Professor assistente do Departamento de Ciência da Informação da

Universidade Federal de São Carlos

E-mail: massao@power.ufscar.br

AMARÍLIO FERREIRA JÚNIOR

Professor adjunto do Departamento de Educação e do

Programa de Pós-Graduação em Educação da

Universidade Federal de São Carlos

E-mail: Ferreira@power.ufscar.br

LEANDRO INNOCENTINI LOPES DE FARIA

Professor adjunto do Departamento de Ciência da Informação da

Universidade Federal de São Carlos

E-mail: leandro@nit.ufscar.br

Recebido em: 12/12/2005

Aceito em: 20/12/2005

(C) ETD. Educação Temática Digital, Campinas, v.7, n.1, p.11-27, dez. 2005 - ISSN: 1676-2592. 